\title{
Postpartum psychosis and sleep disorders: what is the link?
}

Authors: Tyshkevych V, Shkodina A, Kachur R, Boiko D (Mentor), Skrypnikov A (Mentor)

Department Of Psychiatry, Narcology And Medical Psychology, Ukrainian Medical Stomatological Academy, Poltava, Ukraine

DOI: https://doi.org/10.26800/LV-142-supp5-57

\section{Introduction:}

The term "postpartum psychosis" refers to a severe mental illness that begins abruptly shortly after childbirth. The pathophysiology of postpartum psychosis is poorly understood, but factors such as primiparity, difficult labor, genetic predisposition, and hormonal changes have been suggested as etiological factors. It is argued that sleep loss resulting from the interaction of various putative causal factors may be the final common pathway in the development of psychosis in susceptible women.

Aim:

The aim is to investigate the link between sleep disturbances and postpartum psychosis.

\section{Materials \& Methods:}

Searches included MEDLINE, EMBASE, and PUBMED from 2015 to 2020 years. Manual searching was performed on reference lists of included articles. Published primary research in English was included.

\section{Results:}

Postpartum psychosis is one of the few mental disorders with a clear biological trigger: childbirth. Among the factors affecting the likelihood of developing this disease, it is worth noting genetic, hormonal, obstetric risk, drugs, endocrine and immune disorders, as well as sleep and wakefulness disorders. A plausible hypothesis is that sleep disturbance following prolonged labor or nocturnal labor may be associated with the onset of postpartum psychosis. Women with postpartum psychosis had a longer duration of labor and were more likely to have night-time delivery compared to healthy women. Insomnia was the most common and usually the earliest symptom. These preliminary findings indirectly suggest that sleep loss may be the cause of postpartum psychosis in women who are biologically predisposed to the condition.

\section{Conclusion:}

The link between sleep disturbances and postpartum psychosis indicate sleep interventions as a potential low-cost, non-pharmacological prevention and treatment strategy for postpartum mental illness.

Keywords: Childbirth, Psychosis, Sleep disorders 